\title{
Pronator Teres Muscle
}

National Cancer Institute

\section{Source}

National Cancer Institute. Pronator Teres Muscle. NCI Thesaurus. Code C53174.

A muscle of the superficial flexor compartment of the forearm, which originates on both the humeral and ulnar heads and inserts on the body of the radius, that functions to pronate the arm and flex the elbow. 